\title{
XPS study of palladium sensitized nano porous silicon thin film
}

\author{
J KANUNGO, L SELEGÅRD ${ }^{\dagger}$, C VAHLBERG $^{\dagger}$, K UVDAL $^{\dagger}$, H SAHA and S BASU* \\ IC Design and Fabrication Centre, Department of Electronics and Telecommunications Engineering, \\ Jadavpur University, Kolkata 700 032, India \\ ${ }^{\dagger}$ Department of Physics, Chemistry and Biology (IFM), Linköping University, SE-581 83, Linköping, Sweden
}

MS received 27 May 2009

\begin{abstract}
Nano porous silicon (PS) was formed on $p$-type monocrystalline silicon of $2-5 \Omega \mathrm{cm}$ resistivity and (100) orientation by electrochemical anodization method using $\mathrm{HF}$ and ethanol as the electrolytes. High density of surface states, arising due to its nano structure, is responsible for the uncontrolled oxidation in air and for the deterioration of the PS surface with time. To stabilize the material PS surface was modified by a simple and low cost chemical method using $\mathrm{PdCl}_{2}$ solution at room temperature. X-ray photoelectron spectroscopy (XPS) was performed to reveal the chemical composition and the relative concentration of palladium on the nanoporous silicon thin films. An increase of $\mathrm{SiO}_{2}$ formation was observed after $\mathrm{PdCl}_{2}$ treatment and presence of palladium was also detected on the modified surface. $I-V$ characteristics of $\mathrm{Al} / \mathrm{PS}$ junction were studied using two lateral Al contacts and a linear relationship was obtained for Pd modified PS surface. Stability of the contact was studied for a time period of around 30 days and no significant ageing effect could be observed.
\end{abstract}

Keywords. Porous silicon; passivation; palladium; oxidation; XPS.

\section{Introduction}

Porous silicon (PS) is an attractive material due to its extremely large surface to volume ratio, the ease of formation, control of the surface morphology through variation of the formation parameters and its compatibility to silicon IC technology. It has a wide range of applications in photonics and optoelectronics, quantum electronics, silicon-on insulator technology and recently in sensors (Dimitrov 1995; Pavesi and Dubos 1997; Tsamis et al 2002). In spite of all advantages the major barrier against the commercial applications of PS is the temporal degradation of its surface property in oxygen or moisture containing environment and the difficulty in obtaining reliable ohmic contacts (Barillaro et al 2003).

A great deal of research has been focused on the improvement of the quality of PS surface. The high density of dangling bonds and other defect states arising due to its nano structure is mainly responsible for the material instability (Stievenard and Deresmes 1995). Therefore, passivation of the PS surface by some post anodization treatment is necessary (Jeske et al 1995). The need for a stable termination of dangling bonds on the surface of PS has naturally led to the studies of $\mathrm{SiO}_{2}$ as a surface passivation layer. Several groups have reported successful stabilization of the material by furnace oxidation (Tsybeskov et al 1995) or rapid thermal oxidation of PS (Gullis et al 1994; Kanemitsu et al 1994). Though these

*Author for correspondence (sukumar_basu@yahoo.co.uk) processes provide higher quality oxides on the surface with fewer defects but at the same time these high temperature processes may break the PS structure as well. Tomioka et al (2007) reported that the addition of oxidant $\mathrm{H}_{2} \mathrm{O}_{2}$ in $\mathrm{HF}$ solution results in a stable formation of PS layers in a short time. Chen et al (2007) developed an oxidizing technique with ozone for the passivation of PS at room temperature. They achieved a complete replacement of $\mathrm{Si}-\mathrm{H}_{x}$ coverage with $\mathrm{Si}-\mathrm{O}_{x}$ film and $\mathrm{Si}-$ alkyl film. PS prepared with the aid of ozone shows better stability against aging in air.

Controlled oxidation, nitridation, halogenation and polymerization (Lavine et al 1993; Hirschman 1996; Mrazzani 1996; Mandal et al 2006) are a few other methods that have been reported to passivate PS surface. There are reports on coating the surface of PS by the electroless deposition of metals to provide improved stability (Ansager et al 1994).

In the present investigation, we prepared porous silicon by anodization of $p$-Si and modified the surface by a noble metal like Pd using a simple, reliable and low cost chemical method to passivate the defect states. Further, this is a room temperature process and hence the porous structure does not get damaged. The samples were thoroughly characterized by X-ray photoelectron spectroscopy (XPS) to reveal the chemical composition and the relative concentration of palladium on the nanoporous silicon thin films. Currentvoltage measurements were carried out to study the stability of the metal contact to PS after Pd sensitization. 


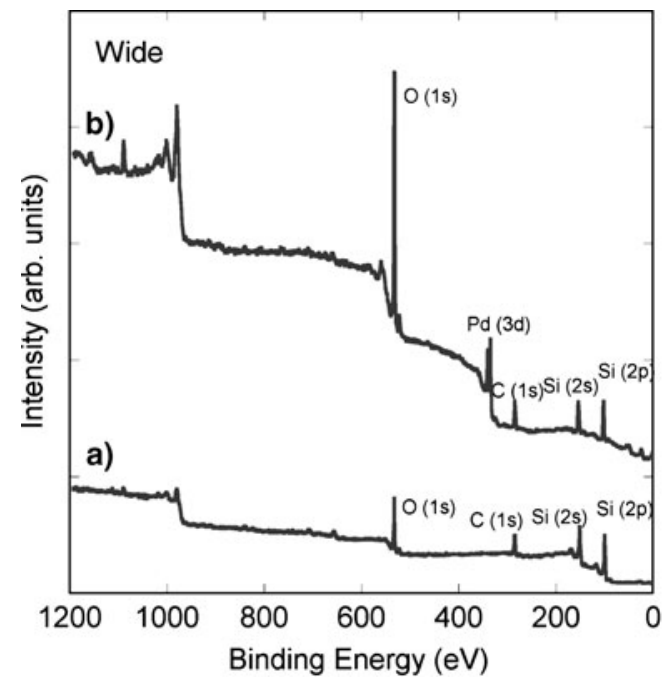

Figure 1. Wide scan XPS spectra of the films: (a) unmodified porous silicon film and (b) palladium modified porous silicon film.

\section{Experimental}

\subsection{Porous silicon formation}

Porous silicon layer was formed by electrochemical anodization of a $p$-type monocrystalline silicon (100) wafer of resistivity $2-5 \Omega \mathrm{cm}$ using a mixture of $\mathrm{HF}$ and ethanol (7:3) as electrolyte. The back of the sample was coated and fired with aluminium paste for making the anodic contact. We applied an etching current density of $10 \mathrm{~mA} / \mathrm{cm}^{2}$ for $40 \mathrm{~min}$. The porosity and thickness of around $55 \%$ and $5 \mu \mathrm{m}$, respectively were measured gravimetrically (Kanungo et al 2006, 2009) using a precision semi-microbalance (290-9842/K, PAG OERLIKONAG CHDIETKON, Switzerland).

\subsection{Modification of porous silicon surface}

PS samples were dipped into $10 \%$ HF solution for $10 \mathrm{~s}$ to remove the native oxide layer to a large extent and were immediately dipped into aqueous $0.01 \mathrm{M} \mathrm{PdCl} 2$ solution (containing 2-3 drops $\mathrm{HCl}$ ) for $5 \mathrm{~s}$. Subsequently, the samples were rinsed gently by DI water, dried and annealed in air at $110^{\circ} \mathrm{C}$ inside an electric oven for $10 \mathrm{~min}$.

The chemical modification steps are cited as follows:

$$
\begin{aligned}
& \mathrm{PdCl}_{2} \rightarrow \mathrm{Pd}^{++}+2 \mathrm{Cl}^{-}, \\
& \mathrm{Pd}^{++} \rightarrow \mathrm{Pd}(\text { Island })+2 h^{+}, \\
& \mathrm{Si}+\mathrm{H}_{2} \mathrm{O}+2 h^{+} \rightarrow \mathrm{Si}(\mathrm{OH})_{2}+2 \mathrm{H}^{+},
\end{aligned}
$$

$$
\begin{aligned}
& \mathrm{Si}(\mathrm{OH})_{2} \rightarrow \mathrm{SiO}_{2}+2 \mathrm{H}^{+}+2 e^{-}, \\
& 2 \mathrm{H}^{+}+2 e^{-} \rightarrow \mathrm{H}_{2}, \\
& 2 \mathrm{Cl}^{-}+2 \mathrm{H}^{+} \rightarrow 2 \mathrm{HCl} .
\end{aligned}
$$

$\mathrm{Pd}^{++}$formed by decomposition of $\mathrm{PdCl}_{2}$ in an aqueous acidic solution is reduced to Pd metal islands by a chemical reduction process (Jeske et al 1995; Kanungo et al 2009) and two holes released. The $h^{+}$oxidizes the PS surface to $\mathrm{SiO}_{2}$.

The XPS measurement was performed on a VG microlab Auger/XPS Spectrometer with a 310-F analyser. The measurements were carried out with $\mathrm{Al} \mathrm{K} \alpha$ photons $(1486.6 \mathrm{eV})$ with a base pressure of around $2 \times 10^{-8} \mathrm{mbar}$ during measurement. Resolution was determined from the full width at half maximum (FWHM) of the $\mathrm{Au}\left(4 f_{7 / 2}\right)$ line in a reference sample and was around $1.5 \mathrm{eV}$ using pass energy of $50 \mathrm{eV}$. The binding energy scale was aligned through the $\mathrm{C} 1 s$ peak at $285 \mathrm{eV}$.

\section{Results}

\subsection{XPS study}

The wide scan XPS spectra for porous Si and Pd modified porous Si are presented in figure 1. Similar line shape of the carbon peaks in both the samples as shown in figures 1 and 2 implies proper calibration of the instrument.

A strong signal from Pd is obtained for the Pd modified porous $\mathrm{Si}$, detected as a spin orbit doublet with $\mathrm{Pd}(3 d)$ peak

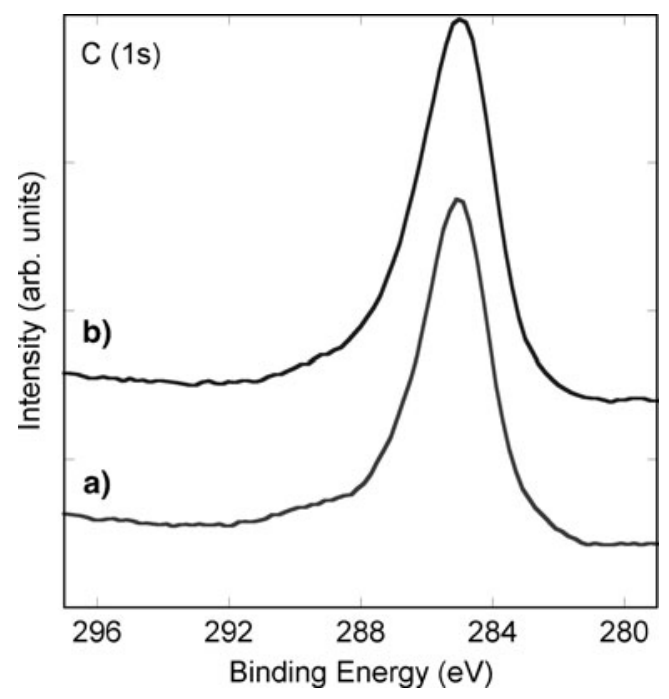

Figure 2. C (1s) XPS core level spectra of the films: (a) unmodified porous silicon film and (b) palladium modified porous silicon film. 
positioned at about $335 \mathrm{eV}$ and $340 \mathrm{eV}$, respectively as shown in figure 3 . No palladium was detected on the porous silicon sample prior to surface modification, as expected.

The $\mathrm{O} 1 s$ spectra in figure 4 clearly indicates that there is a much higher contribution of oxygen in the sample with palladium modified porous silicon, compared to the unmodified one. The $\mathrm{Si} / \mathrm{O}$ ratio is about 0.6 for the palladium modified surface whereas the pure porous silicon surface prior to surface modification has a $\mathrm{Si} / \mathrm{O}$ ratio of $\sim 3 \cdot 4$.

In figure $5 \mathrm{a}$ the main $\mathrm{Si}(2 p)$ peak consists of a spin-orbit coupled doublet ( $\mathrm{Si} 2 p_{3 / 2}$ and Si $2 p_{1 / 2}$ ). The Si $2 p_{3 / 2}$ binding energy peak position is found at $100 \mathrm{eV}$. The split is $\sim 1 \mathrm{eV}$. The peak position indicates that the film consists mainly of pure silicon. There is also one less pronounced binding energy peak at $103 \mathrm{eV}$, consisting of a spin-orbit coupled

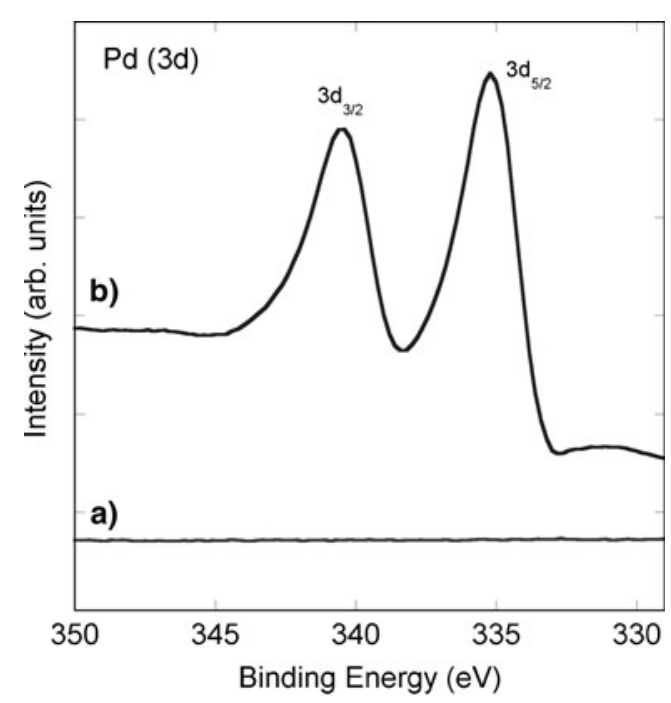

Figure 3. $\operatorname{Pd}(3 d)$ XPS core level spectra of the films: (a) unmodified porous silicon film and (b) palladium modified porous silicon film.

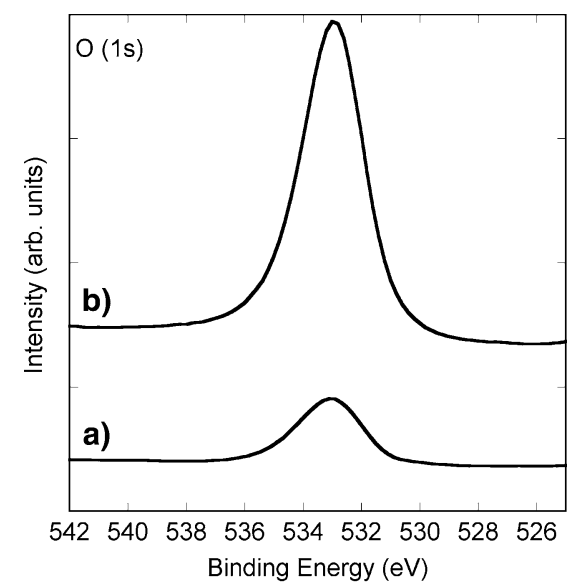

Figure 4. O $(1 s)$ XPS core level spectra of the films: (a) unmodified porous silicon film and (b) palladium modified porous silicon film doublet $\mathrm{Si} 2 p_{3 / 2}$ and $\mathrm{Si} 2 p_{1 / 2}$ corresponding to $\mathrm{SiO}_{2}$. In figure $5 \mathrm{~b}$, the main peak in the spectrum of $\mathrm{Pd}$ modified porous $\mathrm{Si}$ is a strong doublet at around $103 \mathrm{eV}$ in good agreement with $\mathrm{SiO}_{2}$. A small doublet at around $100 \mathrm{eV}$ corresponding to pure silicon is also observed. It indicates that the formation of $\mathrm{SiO}_{2}$ is very much enhanced in presence of $\mathrm{Pd}$ corresponding to the reactions shown above. The $\mathrm{Si} / \mathrm{SiO}_{2}$ ratio on the palladium modified surface is 0.06 and on the porous silicon surface is $5 \cdot 2$, which means that there is around 85 times more $\mathrm{SiO}_{2}$ on the palladium modified surface than on the unmodified porous silicon surface. This is consistent with the observation shown earlier that palladium modified PS surface has been shown to be more oxidized than pristine porous silicon surface.

\subsection{Study of metal/PS contact}

The $I-V$ characteristics were studied using Al lateral contacts (contacts 1 and 2) of dimension $2 \times 2 \mathrm{~mm}$ to PS

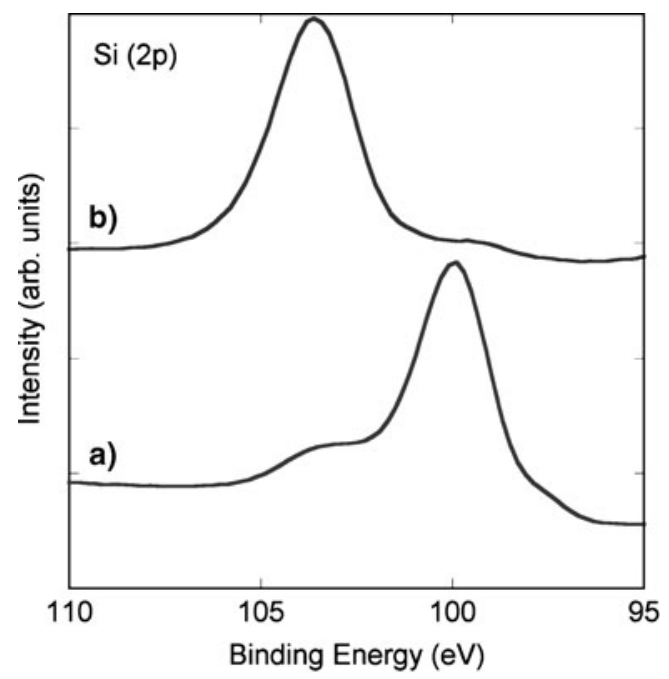

Figure 5. $\mathrm{Si}(2 p)$ XPS core level spectra of the films: (a) unmodified porous silicon film and (b) palladium modified porous silicon film.

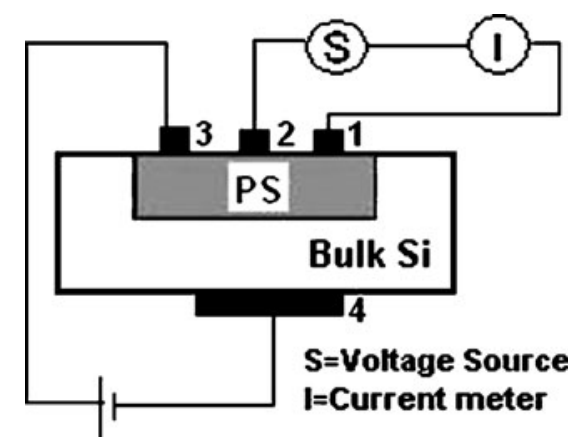

Figure 6. Schematic circuit diagram for $I-V$ measurements. 

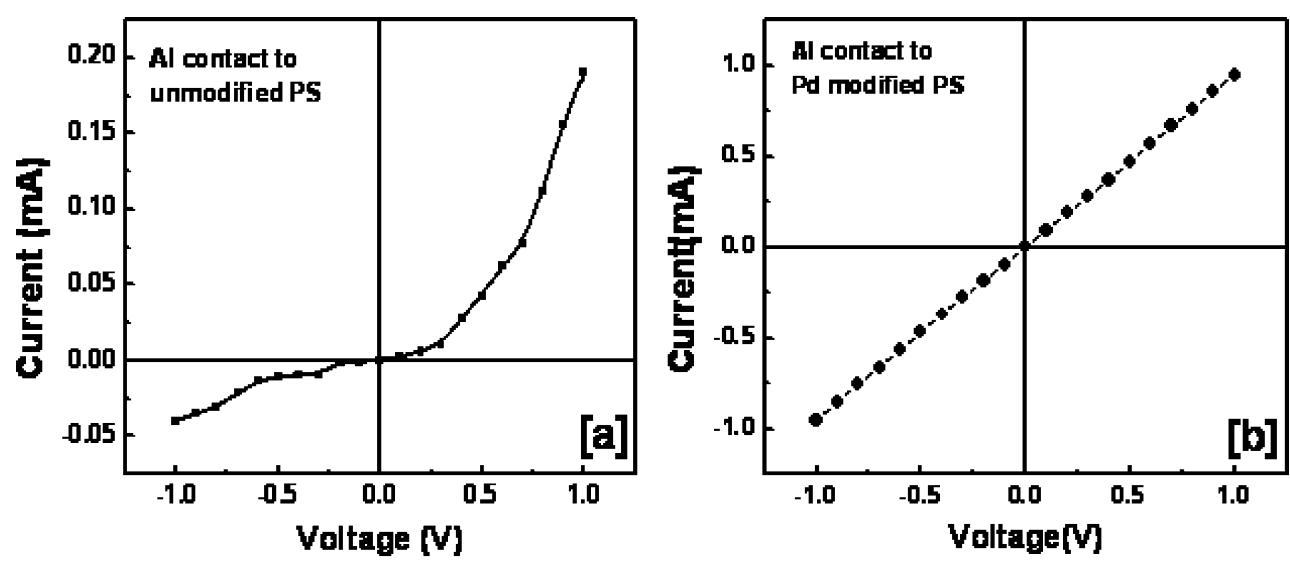

Figure. $7 I-V$ characteristics of (a) unmodified and (b) palladium modified porous silicon using $\mathrm{Al}$ contact.

and separated by a distance of $2 \mathrm{~mm}$ (figure 6) for both unmodified and modified PS surfaces. Since there is a possibility of the current flowing partly through the bulk silicon because of the high resistance of PS, a reverse bias was applied at the PS/p-Si junction (contacts 3 and 4) to keep the current lines confined within the porous silicon layer. All $I-V$ measurements were taken when the PS $/ p-\mathrm{Si}$ junction was completely reverse biased. Detailed measurement condition is reported elsewhere (Kanungo et al 2009). Aluminium is supposed to form ohmic contact with $p$-type porous silicon. Figure 7 clearly demonstrates that the electrical response which is Schottky by nature for unmodified PS becomes ohmic after $\mathrm{PdCl}_{2}$ treatment. This explains the role of $\mathrm{Pd}$ to form $\mathrm{SiO}_{2}$ that passivates the defect states of porous silicon surface and reduces the barrier against the flow of free carriers.

\subsection{Stability of contact}

In order to verify the long-term stability of $\mathrm{Al}$ ohmic contact to PS after modifying the surface with $\mathrm{Pd}, I-V$ measurements were repeated at different intervals of time for a period of around 30 days (figure 8 ) and it was found that the linear $I-V$ curves measured at different time intervals practically overlap signifying a stability of the contact.

\section{Discussion}

After Pd sensitization, as described in § 2.2, Pd clusters are formed on the PS layer, Si surface gets oxidized and a thin layer of $\mathrm{SiO}_{2}$ is formed (Kanungo et al 2009). The $\mathrm{PdCl}_{2}$ treatment enhances oxygen quantity and formation of $\mathrm{SiO}_{2}$ is clearly visible from the XPS results. The Pd cluster and oxidation of PS surface improve the electrical property of $\mathrm{Al} /$ PS contact. The unreliable metal contact to PS is due to unstable porous silicon surface. The high density of surface states on porous silicon, arising due to its nano structure,

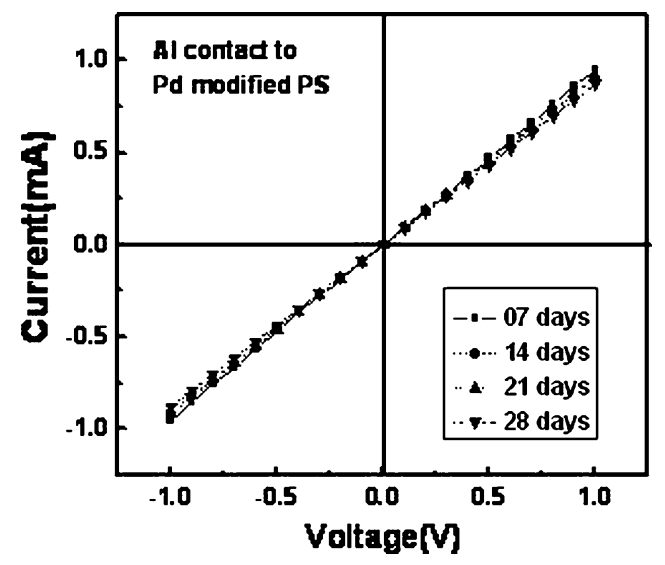

Figure 8. $I-V$ characteristics of $\mathrm{Pd}$ modified porous silicon measured at different intervals of time up to 28 days.

disturbs the stability of the material. Our study indicates that Pd dispersed on the PS surface helps in two ways. The oxidized PS contains less defect states and hence the surface is more stable. Additionally, the dispersed Pd islands help to increase the conductivity as observed from the $I-V$ characteristics of Pd modified PS samples. Electrical property of Pd modified PS is already reported in detail in our earlier communication (Kanungo et al 2009). Since the Pd modification process is carried out at room temperature the possibility of damage of the porous structure is minimized, unlike other methods like furnace oxidations which are already reported.

\section{Conclusions}

The XPS analysis of the Pd modified nano porous silicon surface clearly demonstrates a pronounced increase in the oxidation of porous silicon compared to the unmodified PS. Thin oxide layer protects the PS surface from further deterioration and stabilizes the Al/PS electrical contact as verified by the long-term stability of the current-voltage characteristics. 


\section{Acknowledgements}

The first author thankfully acknowledges the Council of Scientific and Industrial Research (CSIR), Govt. of India, for providing a Senior Research Fellowship. The authors sincerely thank Prof. Anita Lloyd Spetz and Mr Palash Basu for their kind cooperation.

\section{References}

Ansager D, Hilliard J and Nayfeh M H 1994 Appl. Phys. Lett. 64 1141

Barillaro G, Nannini A and Pieri F 2003 Sensors Actuators B93 263

Chen S Y, Huang Y H, Lai H K, Li C and Wang J Y 2007 Solid State Commun. 142358

Dimitrov D B 1995 Phys. Rev. B51 1562

Gullis A G, Canham L T, Williams G M, Smith P W and Dosser O D 1994 J. Appl. Phys. 75493

Hirschman K D 1996 Nature 384338
Jeske M, Schultze J W, Thonissen M and Munder H 1995 Thin Solid Films 25563

Kanemitsu Y, Futagi T, Matsumoto T and Mimura H 1994 Phys. Rev. B49 14732

Kanungo J, Pramanik C, Bandopadhyay S, Gangopadhyay U, Das L, Saha H and Gettens R T T 2006 Semicond. Sci. \& Technol. 21964

Kanungo J, Maji S, Saha H and Basu S 2009 Solid State Electron. 53663

Lavine L M, Sawan S P, Shieh Y T and Bellezza A J 1993 Appl. Phys. Lett. 621099

Mandal N P, Sharma A and Agarwal S C 2006 J. Appl. Phys. 100 024308

Mrazzani V 1996 Thin Solid Films 27632

Pavesi L and Dubos P 1997 Semicond. Sci. \& Technol. 12570

Stievenard D and Deresmes D 1995 Appl. Phys. Lett. 671570

Tomioka S, Miyazaki T and Adachi S 2007 Jpn. J. Appl. Phys. 46 5021

Tsamis C, Tsoura L, Nassiopoulou A G, Travlos A, Salmas C E, Hatzilyberis K A and Ndroutsopoulos G P 2002 IEEE Sensors J. 289

Tsybeskov L, Duttagupta S P and Fauchet P M 1995 Solid State Commun. 95429 